\title{
Engineering the ferromagnetic domain size for optimized imaging of the pinned uncompensated spins in exchange-biased samples by magnetic force microscopy
}

\author{
N. R. Joshi, ${ }^{1}$ S. Özer, ${ }^{1}$ T. V. Ashworth, ${ }^{2}$ P. G. Stickar, ${ }^{3}$ S. Romer, ${ }^{3}$ M. A. Marioni, ${ }^{3, a)}$ and \\ H. J. Hug ${ }^{1,3}$ \\ ${ }^{1}$ Department of Physics, University of Basel, CH-4056 Basel, Switzerland \\ ${ }^{2}$ NanoScan Ltd., CH-8600 Dübendorf, Switzerland \\ ${ }^{3}$ Empa, Swiss Federal Laboratories for Materials Science and Technology, CH-8600 Dübendorf, \\ Switzerland
}

(Received 15 December 2010; accepted 4 February 2011; published online 24 February 2011)

\begin{abstract}
Magnetic force microscopy (MFM) is able to image and quantify patterns of pinned uncompensated spins (UCS) in exchange-biased samples with high spatial resolution and submonolayer spin sensitivity. However, MFM can only detect magnetic moment distributions with spatial wavelengths within a certain range. Samples with large domains, homogeneous, or divergence-free magnetization fields are not accessible to MFM analysis. In this work we discuss the sample structure constraints placed by the requirement to measure UCS at high spatial resolution, and point out a method to engineer the size of the ferromagnetic domains accordingly. () 2011 American Institute of Physics. [doi:10.1063/1.3559228]
\end{abstract}

The spatial distribution and coupling characteristics of pinned uncompensated spins (UCS) in an antiferromagnet $(\mathrm{AF})$ play a key role in the exchange bias $(\mathrm{EB})$ effect of thin film ferromagnet/AF (F/AF) structures. ${ }^{1,2}$ Various experimental methods have been established to map the spatial distribution of UCS. Lateral distributions of UCS can be measured either by x-ray magnetic linear dichroism photoemission electron microscopy (Refs. 3-5) or magnetic force microscopy (MFM). ${ }^{6}$ The former directly accesses an element-specific magnetic state of the sample, whereas the latter measures the stray field emanating from the pinned UCS. $^{7}$ As shown previously ${ }^{7}$ the areal density of the normal (z) magnetic moment from pinned UCS can be deconvolved from the measured MFM data, provided the response function of the MFM tip to stray fields has been calibrated.

Unfortunately, MFM methods are not applicable to arbitrary samples. Special layer and magnetic structures are required, and specific measurement conditions must be warranted. In the following, we discuss these constraints. We restrict our analysis to samples with perpendicular magnetic anisotropy but in-plane magnetic structures can be treated analogously.

The $z$-component of the stray field $\left(H_{z}\right)$ of a sample with a through-thickness up/down magnetic domain pattern is given by:

$$
H_{z}(\mathbf{k}, z)=\frac{M(\mathbf{k}, z)}{2} \exp (-k z)[1-\exp (-k h)]
$$

where $z$ is the distance between the tip and the relevant magnetic surface (called tip-sample distance) and $h$ is the sample thickness, and the relation is expressed in terms of the Fourier coefficients for $\mathbf{k}=\left(k_{x}, k_{y}\right)$. The factors $\exp (-k z)$ and 1 $-\exp (-k h)$ are the so-called distance- and thickness-loss factors, respectively. They account for the fact that components

\footnotetext{
${ }^{\text {a) }}$ Author to whom correspondence should be addressed. Electronic mail: miguel.marioni@empa.ch.
}

with $k h \ll 1$ (large domain sizes) and $k z \gg 1$ (small magnetic features, sharp edges) have a vanishing contribution to the frequency shift measured by MFM. Two important conclusions follow: one, that high spatial resolution can only be attained at small tip-sample distance $z$; and two, MFM will only detect the distribution's stray field wavelengths falling in a specific range. Concerning the detection of pinned UCS with $\approx 10 \mathrm{~nm}$ lateral resolution, the task is, therefore, to engineer the magnetic structures so that the surface to be measured is near the sample surface $(<10 \mathrm{~nm})$ and the $k$ values of the relevant features fall within this range. Note also that MFM registers the total stray field derivative, implying that the fields generated by F-moments and by UCS must be somehow disentangled. This can be accomplished by measuring at F-saturation, ${ }^{6,89}$ since a uniformly magnetized layer produces no stray field.

To study these requirements in more detail we prepared two exchange-coupled structures by dc magnetron sputter deposition at RT, on (001) $\mathrm{Si}$ with native oxide. $\mathrm{S} 1$ is $\mathrm{Pt}(5 \mathrm{~nm}) / \mathrm{AF} / \mathrm{F}^{(\text {top })} / \mathrm{Pt}(2 \mathrm{~nm})$ and $\mathrm{S} 2$ is $\mathrm{Pt}(5 \mathrm{~nm}) /$ $\mathrm{F}^{(\mathrm{btm})} / \mathrm{S}^{(\mathrm{dec})} / \mathrm{AF} / \mathrm{F}^{(\mathrm{top})} / \mathrm{Pt}(2 \mathrm{~nm})$. For $\mathrm{AF}$ we used $1.5 \mathrm{~nm}$ $\mathrm{Co}_{0.8} \mathrm{Cr}_{0.2} \mathrm{O}$, obtained by codeposition of $\mathrm{Co}$ and $\mathrm{Cr}$, annealing at $150{ }^{\circ} \mathrm{C}$ (to segregate $\mathrm{Cr}$ to the grain boundaries) and subsequent oxidation in ambient air. For $\mathrm{F}^{(\mathrm{top})}$, we selected a perpendicular magnetic anisotropy multilayer $[\mathrm{Co}(0.3 \mathrm{~nm}) / \mathrm{Pt}(0.7 \mathrm{~nm})]_{4} / \mathrm{Co}(0.3 \mathrm{~nm})$, deposited at room temperature after oxidation of the $\mathrm{AF}$ layer to circumscribe oxidation to the AF layer. For $\mathrm{F}^{(\mathrm{btm})}$, we selected 10 repeats of the same $\operatorname{Co}(0.3 \mathrm{~nm}) / \mathrm{Pt}(0.7 \mathrm{~nm})$ basic structure, covered with a spacer $\mathrm{S}^{(\mathrm{dec})}=\mathrm{Cu}(2 \mathrm{~nm})$ to exchange-decouple $\mathrm{F}^{(\mathrm{btm})}$ from AF. Limiting the thickness of $\mathrm{F}^{(\text {top })}+$ capping layer to $<5 \mathrm{~nm}$ allows scanning at small tip-to-F/AF-interface distances.

MFM measurements were carried out at $8.3 \mathrm{~K}$ (well below the Neél temperature of the sample, $T_{N}=291 \mathrm{~K}$ ) in UHV (Ref. 10) and in dynamic mode.

After cooling $\mathrm{S} 1$ to $8.3 \mathrm{~K}$ in a $1 \mathrm{~T}$ field, vibrating sample magnetometry revealed an EB-field $\mu_{0} H_{\mathrm{ex}}=-296.4 \mathrm{mT}$ and 

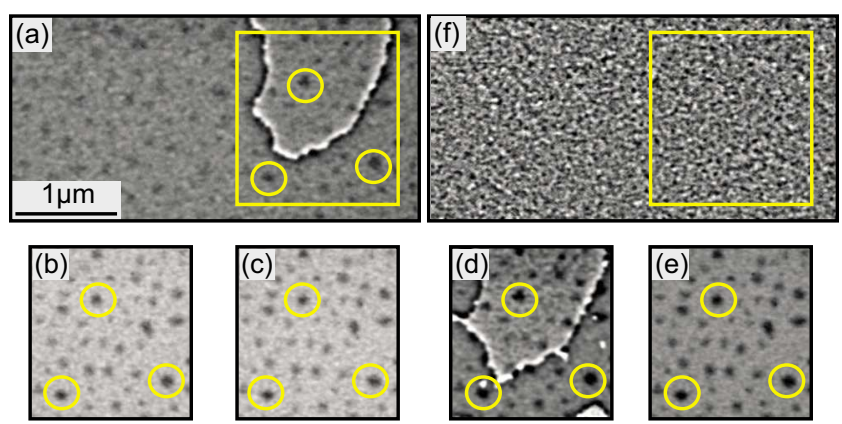

FIG. 1. (Color online) MFM data: (a) after zero-field cooling to $8.3 \mathrm{~K}$; square outline: area of insets (b)-(e). Circles: some topographical features. (b) Inset: saturation in $1 \mathrm{~T}$; (c) inset: saturation in $-1 \mathrm{~T}$; (d) inset: recovery of (a) at $+260 \mathrm{mT}$ after (c); (e) inset: semisum $(\mathrm{b}+\mathrm{c}) / 2$ : topography; and (f) semidifference $(b-c) / 2$ : pinned UCS.

coercive fields of $\mu_{0} H_{\mathrm{c}}^{+}=+296.3 \mathrm{mT}, \mu_{0} H_{\mathrm{c}}^{-}=-592.7 \mathrm{mT}$. Microscopically, the magnetic structures of S1 were studied by MFM directly after cooling in zero applied field. Figure 1(a) shows part of the MFM data. A bubble domain at the top of the initially measured $4 \mu \mathrm{m} \times 2 \mu \mathrm{m}$ image is apparent. The large domain size and the small F-layer thickness result in a strong stray field gradient at the domain wall but only a weak stray field in the domain center. Accordingly there is a pronounced black/white contrast only near the domain walls (domain wall contrast). Inside the domains a granular contrast and some isolated darker areas are visible [circles in Figs. 1(a)-1(e)]. The granularity and the darker areas mainly stem from the topography of the sample, which gives rise to variations in the van der Waals force.

To distinguish pinned UCS from other sources of MFM contrast, we apply saturating fields of $\pm 1 \mathrm{~T}$ perpendicular to the sample. The corresponding MFM images obtained at $z$ $=10 \pm 3 \mathrm{~nm}$ [Figs. 1(b) and 1(c)] of the saturated F-layer present the same granular contrast and the isolated darker areas as Fig. 1(a). A domain structure corresponding to pinned UCS is not visible. Recall that at $1 \lesssim z \lesssim 10 \mathrm{~nm}$ the contributions of van der Waals forces to the MFM contrast will dominate over magnetic forces from pinned UCS (presumed few). However, the van der Waals interaction is independent of the magnetic field (it depends on the tip-sample distance), opening a way to distinguishing them from the magnetic forces we are interested in. To that end consider the sign of the interactions at $\pm 1 \mathrm{~T}$, given that the tip magnetization switches into alignment with fields $|H| \gtrsim 50 \mathrm{mT}$. Since both the saturated F moments and the rotating UCS will align parallel to the $\pm 1 \mathrm{~T}$ field, their interaction with the MFM tip will be equivalent. In contrast, pinned UCS retain their orientation even at $1 \mathrm{~T}$ field levels $( \pm 7 \mathrm{~T}$ fail to reorient them $^{9}$ ), so the sign of their interaction with the tip will change sign with the applied $\pm 1 \mathrm{~T}$ fields.

Calculating half of the sum of MFM data acquired in $+1 \mathrm{~T}$ [Fig. 1(b)] and $-1 \mathrm{~T}$ [Fig. 1(c)] then reflects the topography-induced frequency shift [Fig. 1(e)]. Conversely, one half of the difference shows the contrast caused by the pinned UCS [Fig. 1(f)]. The black spots in Fig. 1(e) (examples pointed out by circles) occur when the tip scans over particles on the surface of the sample. These reduce the local tip-sample distance causing a more attractive van der Waals force and thereby a more negative frequency shift.

Figure 1(f) displays a granularity on a fine scale which does neither correspond to the topography-induced black
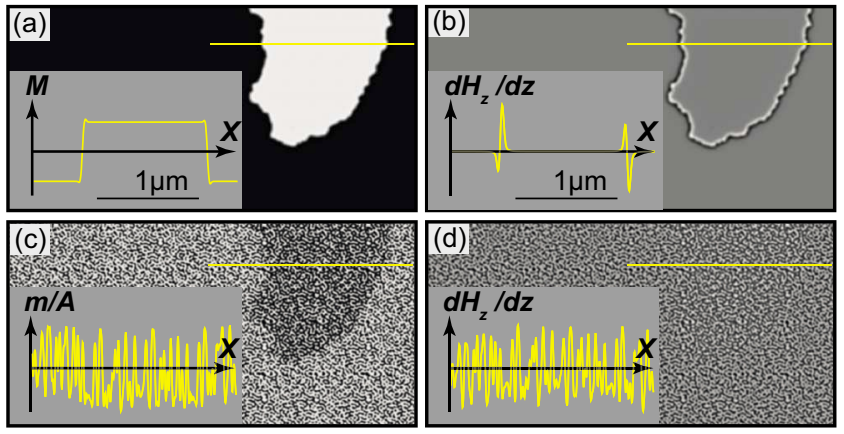

FIG. 2. (Color online) (a) Model F-domain pattern. (b) Domain wall contrast in calculated $d H_{z} / d z$-pattern. (c) Model UCS pattern. (d) Corresponding $d H_{z} / d z$-pattern.

spots in Fig. 1(e) nor correlate with the F-pattern immediately after zero-field cooling in Fig. 1(a). It is, therefore, surprising that in a field of $+260 \mathrm{mT}[$ Fig. 1(d)] applied after saturation in $-1 \mathrm{~T}$ [Fig. 1(c)] a F-domain pattern matching Fig. 1(a) is obtained, given that the coercivity is only $500 \mathrm{mT}$ and recovery is not caused by reversal domains at $-1 \mathrm{~T}^{7}$ In fact, it points out the existence of an imprinted UCS pattern governing domain nucleation and growth, and leading to recovery but failing to register in the MFM signal.

To see why the pinned UCS are not visible in Fig. 1(f), we analyze in more detail the stray field derivative $d H_{z} / d z$ to which MFM is sensitive. The F pattern inducing the pinned UCS in S1 can be modeled by a bivalued up/down magnetization distribution, ideally as in Fig. 2(a). It results in the stray field derivative pattern shown in Fig. 2(b), which is a good match to Fig. 1(a). As expected for domain sizes much larger than the thickness of the F-layer it shows contrast (only) near the domain walls. In terms of transferring magnetization spatial wavelengths to the image as per Eq. (1), Fig. 2(b) can be said to have strongly dampened long spatial wavelengths.

The pinned UCS produced by this F pattern are known to couple antiparallel to the F spins on average, and display a granularity of the order of the grain size, ${ }^{7}$ about $10 \mathrm{~nm}$. They can be described as the sum of two parts: one, a pattern identical to Fig. 2(a) with an amplitude of $\pm 20 \%$ of a $\mathrm{CoO}$ monolayer and opposite sign (antiparallel average coupling). Two, a random up/down magnetic moment distribution with an amplitude of $\pm 100 \%$ of a $\mathrm{CoO}$ monolayer, and granularity of $10 \mathrm{~nm}$. The total pinned UCS magnetic moment distribution is depicted in Fig. 2(c). Only these pinned UCS generate contrast when the $\mathrm{F}$ is saturated [as in Fig. 1(f)]. Specifically, the $d H_{z} / d z$ map from Fig. 2(c) is calculated in Fig. 2(d). Thus, the UCS contrast is seen to comprise a weak domain wall contrast from the UCS (i.e., formally the same as Fig. 2(b) but with weaker and inverted contrast) and a dominating "noiselike" contrast from the grain-size scale fluctuations in pinned UCS. The correlation with the F-domain pattern of Fig. 2(a) essentially disappeared. From this, we conclude that for the detection of pinned UCS it is inconvenient to have domain wall contrast in the F. Domain contrast is preferable, because the spatial frequencies carrying the information of the average pinned UCS density are retained, so the latter are visible despite amplitude fluctuations.

To obtain domain contrast we need smaller domains, and hence a larger thickness of the F-layer. This requirement is at 


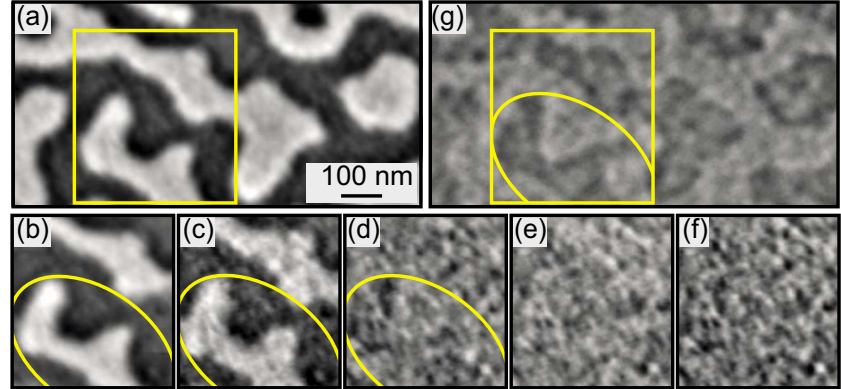

FIG. 3. (Color online) $4 \mu \mathrm{m} \times 2 \mu \mathrm{m}$ MFM data from S2. (a) Domain contrast after zero-field cooling to $8.3 \mathrm{~K}$. Square outline: inset area. (b) 120 $\mathrm{mT}$ inset. (c) $190 \mathrm{mT}$ inset: $\mathrm{F}^{\mathrm{btm}}$ is saturated. (d) $500 \mathrm{mT}$ inset: $\mathrm{F}^{\mathrm{btm}}$ and $\mathrm{F}^{\text {top }}$ are saturated. A granular contrast dominates but the imprinted UCS pattern is visible ("F"-shaped domain in yellow ellipse). (e) $-500 \mathrm{mT}$ : saturation. (f) semisum inset $(d+e) / 2$ : topography signal. $(g)$ Semidifference $(d-e) / 2$ : pinned UCS.

odds with the constraint from high resolution MFM imaging that the F-layer be thin, to allow keeping the tip-to-F/AFinterface distance small. A sample $\mathrm{S} 2$ with a basic structure $\mathrm{F}^{(\mathrm{btm})} / \mathrm{S}^{(\mathrm{dec})} / \mathrm{AF} / \mathrm{F}^{(\mathrm{top})}$ was fabricated as a way out of this quandary. $\mathrm{F}^{(\mathrm{btm})}+\mathrm{F}^{(\mathrm{top})}$ have a thickness of $15 \mathrm{~nm}$, which sets the domain size to $100-200 \mathrm{~nm}$. In the as-grown state the domains will penetrate through both F-layers. The $2.2 \mathrm{~nm}$ thick $\mathrm{Cu}$ layer breaks the magnetic exchange between the thick $\mathrm{F}^{(\mathrm{btm})}$ and the AF. Accordingly $\mathrm{F}^{(\mathrm{btm})}$ was found to switch symmetrically with a coercive field $\mu_{0} H=54 \mathrm{mT}$, i.e., it is not exchange-biased. For the thin $\mathrm{F}^{(\text {top })}$ an exchange field $H_{\mathrm{ex}}=-124.5 \mathrm{mT}$ and coercive fields of $-405 \mathrm{mT}$ and $+156 \mathrm{mT}$ are found.

Figure 3(a) shows the F-domain pattern from S2 measured after zero-field cooling. The domains are about an order of magnitude smaller than in S1. In contrast to S1 [Fig. 1(a)] the domains from $S 2$ generate a pronounced domain contrast [Fig. 3(a)]. Inside the domains a weak granular contrast is visible. If the field is raised to $120 \mathrm{mT}$ the $\mathrm{F}^{(\mathrm{btm})}$ domains start to vanish but the $\mathrm{F}^{(\mathrm{top})}$-domains are retained. At $190 \mathrm{mT} \mathrm{F}^{(\mathrm{btm})}$ is saturated [Fig. 3(c)], while the structure of $\mathrm{F}^{\text {(top) }}$-domains remains unchanged [see panel (c) to (a)], albeit with diminished domain contrast and hence more prominent granularity. Again, we apply saturating fields in the positive and negative direction perpendicular to the sample. The corresponding MFM images [Figs. 3(d) and 3(e)] reveal a faint pattern of pinned UCS dominated by a granular contrast. For example the "F"-shaped up-domain inside the ellipse imprints a corresponding " $F$ "-shaped pattern of pinned down-UCS in Fig. 3(d).

As before, we compute the semisum and semidifference of the MFM data acquired in saturation, $+500 \mathrm{mT}$ [Fig. 3(d)] and $-500 \mathrm{mT}$ [Fig. 3(e)] in this case. The former yields the topography-induced frequency shift [Fig. 3(f)], whereas the latter gives the contrast caused by the pinned UCS [Fig. $3(\mathrm{~g})]$. Consistent with previous work, ${ }^{6-9}$ we find the $\mathrm{F}$ domain pattern imprints a corresponding antiparallel pattern of pinned UCS in the AF. The pattern of pinned UCS is clearly visible. This MFM frequency-shift data is now in a form that can be deconvolved to obtain the areal density of the $z$-component of the all pinned magnetic moments in the AF layer.

In conclusion, we have shown that the sample's layering and the spatial wavelengths of its magnetic structure condition the measurement of pinned UCS by MFM in an exchange-biased perpendicular structure. Since the long spatial wavelength magnetization variations cannot be detected by the MFM, the average values of the pinned UCS can be detected easily only in domain contrast conditions. For these to be given concurrently with small tip-sample distances, as required by high spatial resolution MFM, the F-layer can be split in two parts. One part stabilizes small domains magnetostatically but is exchange decoupled from the AF, far from the MFM tip. The other part induces the pinned UCS in the $\mathrm{AF}$ (and is thus exchange-biased), and can be kept thin and close to the MFM tip.

In samples with in-plane magnetic anisotropy, where domain sizes are typically greater than $1 \mu \mathrm{m}$, the discussed addition of a thick $\mathrm{F}^{\mathrm{btm}}$ underlayer would also be useful. It is expected that the narrow perpendicular domains generated induce corresponding in-plane domains in the thin $\mathrm{F}^{\text {top }}$ layer, enabling the imaging of pinned UCS in these systems.

The financial support of the Swiss National Science Foundation (Grant Nos. 200021-117970 and 200021113821/1, and the NCCR NanoScale Science of the University of Basel is hereby gratefully acknowledged.

${ }^{1}$ F. Offi, W. Kuch, L. I. Chelaru, M. Kotsugi, and J. Kirschner, J. Magn. Magn. Mater. 261, 1 (2003).

${ }^{2}$ M. Tsunoda, T. Nakamura, M. Naka, S. Yoshitaki, and C. Mitsumata, Appl. Phys. Lett. 89, 172501 (2006).

${ }^{3}$ F. Nolting, A. Scholl, J. Stöhr, J. W. Seo, J. Fompeyrine, H. Siegwart, J.-P. Locquet, S. Anders, J. Lüning, E. F. M. F. Toney, M. R. Scheinfein, and H. A. Padmore, Nature (London) 405, 767 (2000).

${ }^{4}$ T. Eimüller, T. Kato, T. Mizuno, S. Tsunashima, C. Quitmann, T. Ramsvik, S. Iwata, and G. Schutz, Appl. Phys. Lett. 85, 2310 (2004).

${ }^{5}$ Y. Du, G. Pan, R. Moate, H. Ohldag, A. Kovacs, and A. Kohn, Appl. Phys. Lett. 96, 222503 (2010).

${ }^{6}$ P. Kappenberger, S. Martin, Y. Pellmont, H. J. Hug, J. B. Kortright, O. Hellwig, and E. E. Fullerton, Phys. Rev. Lett. 91, 267202 (2003).

${ }^{7}$ I. Schmid, M. A. Marioni, P. Kappenberger, S. Romer, M. ParlinskaWojtan, H. J. Hug, O. Hellwig, M. J. Carey, and E. E. Fullerton, Phys. Rev. Lett. 105, 197201 (2010).

${ }^{8}$ I. Schmid, P. Kappenberger, O. Hellwig, M. J. Carey, E. E. Fullerton, and H. J. Hug, EPL 81, 17001 (2008).

${ }^{9}$ P. Kappenberger, I. Schmid, and H. Hug, Adv. Eng. Mater. 7, 332 (2005).

${ }^{10}$ H. J. Hug, B. Stiefel, P. J. A. van Schendel, A. Moser, S. Martin, and H.-J. Güntherodt, Rev. Sci. Instrum. 70, 3625 (1999). 